\title{
A Comparative Study on Breast Milk Feeding and Formula Milk Feeding in Infants With Congenital Heart Disease After Surgery: A Retrospective Study
}

\author{
Xian-Rong Yu, MM, ${ }^{1,2}$ Ning Xu, MM, ${ }^{1,2}$ Shu-Ting Huang, MM, ${ }^{1,2}$ Ze-Wei Lin, MM, ${ }^{1,2}$ \\ Zeng-Chun Wang, MD, ${ }^{1,2,3}$ Hua Cao, MD, ${ }^{1,2}$ Qiang Chen, $\mathrm{MD}^{1,2,3}$ \\ ${ }^{1}$ Department of Cardiac Surgery, Fujian Maternity and Child Health Hospital, Affiliated Hospital of Fujian Medical University, \\ Fuzhou, China; ${ }^{2}$ Fujian Key Laboratory of Women and Children's Critical Diseases Research, Fujian Maternity and Child Health \\ Hospital, Fuzhou, China; ${ }^{3}$ Department of Cardiovascular Surgery, Union Hospital, Fujian Medical University, Fuzhou, China
}

\section{ABSTRACT}

Objective: To explore the effects of breast milk feeding and formula milk feeding on infants after cardiac surgery in the cardiac intensive care unit (ICU).

Methods: Infants who underwent cardiac surgery in our ICU were divided into two groups, according to feeding type. Breast milk feeding and formula milk feeding were separately implemented in the two groups, and the remaining treatment regimens were the same. The related clinical data and feeding effects were recorded and compared.

Results: The prealbumin $(147.3 \pm 15.2$ versus $121.5 \pm$ $18.3 \mathrm{mg} / \mathrm{L})$ and albumin $(46.4 \pm 4.2$ versus $40.5 \pm 5.1 \mathrm{~g} / \mathrm{L})$ levels in the breast milk feeding group were better than those in the formula milk feeding group $(P<.05)$. Infants in the breast milk feeding group achieved a better total enteral nutrition time $(3.0 \pm 1.2$ versus $5.2 \pm 2.1 \mathrm{~d})$, average daily weight gain $(19.0 \pm 3.4$ versus $14.4 \pm 2.3 \mathrm{~g} / \mathrm{kg} \cdot \mathrm{d})$, length of ICU stay $(6.0 \pm 2.2$ versus $8.1 \pm 2.9 \mathrm{~d})$ and length of hospital stay $(13.9 \pm 4.2$ versus $17.8 \pm 5.6 \mathrm{~d})$ than those in the formula milk feeding group $(P<.05)$. The incidence of complications such as feeding intolerance, anemia, dyspeptic diarrhea, and nosocomial infection was lower in the breast milk feeding group than in the formula milk feeding group $(P<0.05)$.

Conclusion: Breast milk feeding has a definite nutritional effect on infants after cardiac surgery. It is better than formula milk feeding, making it worthy of popularization and application.

\section{INTRODUCTION}

Studies have shown that congenital heart disease (CHD) is one of the most common types of congenital malformations,

Received August 16, 2020; accepted September 9, 2020.

Correspondence: Qiang Chen, MD, Department of Cardiac Surgery, Fujian Maternity and Child Health Hospital, Affiliated Hospital of Fujian Medical University, Fuzhou, China; Fujian Key Laboratory of Women and Children's Critical Diseases Research, Fujian Maternity and Child Health Hospital, Fuzhou, China; Department of Cardiovascular Surgery, Union Hospital, Fujian Medical University, Fuzbou, China (e-mail: chenqiang2228@163.com). accounting for approximately $28 \%$ of various congenital malformations [San Agustin 2016]. Severe CHD and pulmonary hypertension may endanger the lives of some infants. Surgical repair in infancy is the main intervention for these patients with CHD, who usually have associated malnutrition and growth retardation [Yun 2011; El-Alameey 2015]. With improvements in surgical techniques and perioperative management strategies, the operative success rate of CHD in infancy is increasing, although there are still some postoperative complications. Due to the lower muscle mass and fat percentage, the limited energy and protein reserves, and the higher metabolism and increased energy and protein requirements after cardiac surgery, infantile patients are particularly vulnerable to nutrition-related problems [Boban 2013; Prieto 2011]. Therefore, postoperative nutritional support is another important aspect of the clinical management of infantile patients after surgical repair. Breast milk has a unique composition and good immune protection, which can effectively promote the growth and development of infants and accelerate the recovery of diseases [Andreas 2015; Hassiotou 2013; Hinde 2012]. By consulting a large number of articles, few reports are found on the nutritional status and nutrition interventions of infants undergoing cardiac surgical correction. In this paper, a retrospective study was conducted to investigate the effect of breast milk feeding and formula milk feeding in infants who underwent cardiac surgical correction.

\section{MATERIALS AND METHODS}

The sample size was determined by PASS 15.0. The alpha value was set to 0.05 , and the power was 0.90 . According to the calculation, the minimum sample size was 48 patients. Considering a $15 \%$ dropout rate, 28 patients were included in both groups. This was a retrospective study.

A total of 56 infants who underwent congenital heart surgery at our cardiac department between March 2020 and July 2020 were included. All the patients were divided into a research group (breast milk feeding group) and a control group (formula milk feeding group). The inclusion criteria were as follows: (1) all infants were diagnosed with CHD by transthoracic echocardiography or CT examination before 
Table 1. Comparison of the general data between the two groups

\begin{tabular}{lccc}
\hline & $\begin{array}{c}\text { Breast milk } \\
\text { group }\end{array}$ & $\begin{array}{c}\text { Formula milk } \\
\text { group }\end{array}$ & $P$ \\
\hline Age (months) & $1.8 \pm 1.1$ & $1.7 \pm 1.0$ & .576 \\
Weight $(\mathrm{kg})$ & $3.9 \pm 1.5$ & $4.0 \pm 1.1$ & .652 \\
Disease & & & \\
Ventricular septal defect & 20 & 21 & .934 \\
Pulmonary stenosis & 3 & 4 & \\
Coarctation of aorta & 2 & 1 & \\
Total anomalous pulmonary & 2 & 1 & \\
venous connection & 1 & 1 & \\
Interrupted aortic arch & $137.2 \pm 18.7$ & $133.2 \pm 15.8$ & .712 \\
Prealbumin (mg/L) & $33.5 \pm 4.1$ & $32.5 \pm 4.6$ & .624 \\
Albumin (g/L) & $3.6 \pm 1.3$ & $3.5 \pm 1.5$ & .731 \\
Operation time (h) & $1.8 \pm 0.8$ & $1.6 \pm 0.7$ & .763 \\
Cardiopulmonary bypass time (h) & & & \\
Aortic cross-clamping time $(\mathrm{h})$ & $0.9 \pm 0.4$ & $0.8 \pm 0.3$ & .874
\end{tabular}

the operation, and there were no other associated organ diseases or other surgical history; (2) all patients were treated surgically by the same team of surgeons, postoperative echocardiography indicated satisfactory correction of intracardiac malformation, postoperative hemodynamics were stable, endotracheal intubation was successfully removed, and the basic medical treatment was the same for both groups; and (3) family members were informed about the research and voluntarily participated. The exclusion criteria were as follows: (1) combined with severe gastrointestinal abnormalities or metabolic diseases; (2) patients with complex congenital heart disease requiring staged surgery; (3) chylothorax after the operation; (4) milk protein allergy; and (5) family members refused to participate in the study.

The study was approved by the ethics committee of our hospital, and consent from the guardian of each research subject was obtained. Patient general clinical information is listed in Table 1. All patients underwent surgical correction under cardiopulmonary bypass and achieved satisfactory clinical results. Because our hospital is located in the city and some patients were from remote rural areas, these families could not provide breast milk in a timely manner. These infants had to receive formula milk feeding and were assigned to the control group. Families who could provide breast milk feeding were assigned to the research group.

A medical sterile milk cup was used to make the formula milk, which was adjusted to liquid, according to the instructions. Breast milk was timely collected by the mother of the patient and refrigerated in bags. When feeding was needed, the refrigerated milk was thawed and heated with $40^{\circ} \mathrm{C}$ warm water in the milk warmer. All the infants had gastric tubes inserted through the nasal cavity after surgery. Regardless of whether tracheal intubation was removed, the two groups of
Table 2. Comparison of the postoperative data between the two groups

\begin{tabular}{lccc}
\hline & $\begin{array}{c}\text { Breast milk } \\
\text { group }\end{array}$ & $\begin{array}{c}\text { Formula } \\
\text { milk group }\end{array}$ & $P$ \\
\hline Mechanical ventilation duration (d) & $4.1 \pm 1.2$ & $4.5 \pm 1.4$ & .248 \\
Length of intensive care unit stay (d) & $6.0 \pm 2.2$ & $8.1 \pm 2.9$ & .045 \\
Start feeding time (h) & $27.8 \pm 7.4$ & $50.4 \pm 13.7$ & .022 \\
Total enteral nutrition time (d) & $3.0 \pm 1.2$ & $5.2 \pm 2.1$ & .038 \\
Average daily weight gain (g/kg.d) & $19.0 \pm 3.4$ & $14.4 \pm 2.3$ & .041 \\
Length of hospital stay (d) & $13.9 \pm 4.2$ & $17.8 \pm 5.6$ & .037 \\
Pre-albumin (mg/L) & $147.3 \pm 15.2$ & $121.5 \pm 18.3$ & .029 \\
Albumin (g/L) & $46.4 \pm 4.2$ & $40.5 \pm 5.1$ & .034
\end{tabular}

infants began to feed on the second to fourth day after surgery when bowel sounds were detected by abdominal auscultation. After confirming the amount of feeding, these two kinds of milk were separately loaded into syringes and injected into the stomach through the gastric tube by an infusion pump with a uniform velocity. Other routine postoperative treatments, including cardiotonic, diuretic, and anti-infective treatments as well as radiation platform warming, were routinely performed in both groups. The contents of the stomach were extracted before feeding, and the amount of residual milk was observed. If the amount of residual milk was less than $10 \%$ of the total feeding amount, the gastrointestinal function was considered good. Therefore, the total amount of milk to be fed during the next feeding time would increase the amount of residual milk. All patients were fed every $2 \sim 3 \mathrm{~h}$, the initial feeding amount was $0.5-1 \mathrm{ml} /(\mathrm{kg} \cdot \mathrm{d})$, and the daily feeding amount gradually was increased, according to the specific situation of the patients. If feeding tolerance was achieved, the daily feeding amount was set as $100-120 \mathrm{ml} /(\mathrm{kg} \cdot \mathrm{d})$. On this basis, all patients received intravenous nutrition to supplement caloric deficiency after inadequate gastrointestinal feeding, including glucose, fat emulsions, amino acids, vitamins, and electrolytes. When the amount of feeding reached the set goal of total intestinal feeding, intravenous nutritional support gradually was removed. After endotracheal intubation was removed, the patient received oral feeding or continued nasal feeding, as appropriate.

The total enteral feeding time, average daily weight gain of the infants, and length of ICU and hospital stays in the two patient groups were compared. The preoperative albumin and prealbumin values of the two groups were recorded and monitored again before discharge. The incidence of complications, such as feeding intolerance, anemia, and nosocomial infections during the feeding process, were assessed. The color and quantity of gastric remnants were evaluated by gastric tube aspiration before each feeding. The diagnostic criteria for feeding intolerance were as follows: The infants had symptoms, such as gastric retention and vomiting, and gastric retention was more than $50 \%$ of the previous total feeding amount. Physical examination revealed increased bowel 
Table 3. Comparison of the complications between the two groups

\begin{tabular}{lccc}
\hline & Breast milk group & Formula milk group & $P$ \\
\hline Feeding intolerance & 1 & 7 & .022 \\
Anemia & 1 & 6 & .043 \\
Hypoglycemia & 1 & 2 & .639 \\
Hypocalcemia & 3 & 4 & .686 \\
Dyspeptic diarrhea & 0 & 4 & .038 \\
Nosocomial infection & 1 & 6 & .043
\end{tabular}

sounds and abnormal abdominal tenderness. The diagnostic criteria for dyspeptic diarrhea were as follows: defecation multiple times a day, mostly in the morning or after feeding, and mushy and watery stool, with a pungent odor.

SPSS 25.0 (IBM Corp., Armonk, NY) statistical software was used to analyze the data. The count data were expressed as a percentage, and the measurement data were expressed as $\mathrm{x} \pm \mathrm{s}$. All data were tested for a normal distribution, and data that conformed to a normal distribution were evaluated by paired $\mathrm{T}$ tests. Sex, disease, intolerance rate of feeding, anemia, hypoglycemia, hypocalcemia, dyspeptic diarrhea and nosocomial infections were evaluated by the chi-square test. $P<.05$ was considered statistically significant.

\section{RESULTS}

As shown in Table 1, there was no significant difference in age, sex, weight, disease, preoperative prealbumin level, preoperative albumin level, operation time, cardiopulmonary bypass time or aortic cross-clamping time between the two groups. However, the prealbumin $(147.3 \pm 15.2$ versus 121.5 $\pm 18.3 \mathrm{mg} / \mathrm{L})$ and albumin levels $(46.4 \pm 4.2$ versus $40.5 \pm$ $5.1 \mathrm{~g} / \mathrm{L}$ ) in the breast milk feeding group were significantly better than those in the formula milk feeding group before discharge $(P<.05)$. Compared with the formula milk feeding group, the breast milk feeding group showed significant differences in the time of achieved total enteral nutrition $(3.0 \pm$ 1.2 versus $5.2 \pm 2.1 \mathrm{~d})$, average daily weight gain $(19.0 \pm 3.4$ versus $14.4 \pm 2.3 \mathrm{~g} / \mathrm{kg} \cdot \mathrm{d})$, length of ICU stay $(6.0 \pm 2.2$ versus $8.1 \pm 2.9 \mathrm{~d})$, and length of hospital stay $(13.9 \pm 4.2$ versus 17.8 $\pm 5.6 \mathrm{~d})(P<.05)$ (Table 2$)$. As shown in Table 3 , the incidence of complications, such as feeding intolerance, anemia, dyspeptic diarrhea, and nosocomial infection, was significantly lower in the breast milk feeding group than in the formula milk feeding group $(P<.05)$, and there was no significant difference in hypoglycemia or hypocalcemia between the two groups $(P>.05)$ (Table 3$)$.

\section{DISCUSSION}

CHD is one of the most common congenital defects, and the prevalence of CHD at birth is six to 13 cases per
1,000 live births [Zaidi 2017; Liu 2013; Khoshnood 2012; Wren 2012]. Patients' symptoms and signs vary with the type of heart disease. Symptoms can include shortness of breath, cyanosis, slow weight gain, and so on. Some serious cases may cause other related complications, which can be life-threatening. In the past few decades, there has been significant progress in the surgical techniques and perioperative management strategies for CHD [Goldberg 2015]. As the spectrum of disease has changed, clinicians now face patients who are younger, more critical, and more complicated. An increasing number of patients with CHD need surgical correction in infancy. Infancy is an important stage of development, and infant metabolism is characterized by not only meeting postoperative energy consumption but also meeting the needs of growth and development [Glavin 2014]. Higher postoperative energy consumption results in higher requirements for nutritional support. The relationship between CHD and malnutrition widely has been studied, especially in developing countries, where the prevalence rate ranges from $27 \%$ to $90.4 \%$ [Venugopalan 2001]. Malnutrition in infants with CHD after surgery may be related to the following reasons: fatigue associated with the eating process, which might reduce calorie intake; intestinal malabsorption secondary to possible low cardiac output postoperatively; and increased energy consumption secondary to increased use of respiratory muscles [Radman 2014]. Postoperative nutritional status has been associated with clinical outcomes, such as mortality, infection incidence, and ICU and hospital stay length. Therefore, the nutritional support of these infants after cardiac surgery is very important to maintain organ function and prevent cardiovascular dysfunction. The purpose of this study was to compare the effects of breast milk feeding and formula milk feeding on infants with CHD after surgical correction.

Kreissl et al compared the effects of using breast milk or formula milk for enteral feeding in preterm infants on the time to total enteral feeding and complications, and the results showed that breast milk instead of formula milk fed to preterm infants could shorten the time of total enteral feeding and reduce the incidence of septic retinopathy in premature infants [Kreissl 2017]. The results of our study also were consistent with these conclusions. Breast milk feeding in infants after cardiac surgery has several advantages. First, there are a variety of antioxidant enzymes, digestive enzymes, hormones and growth factors in breast milk, which have a good effect on the gastrointestinal tract and enhance digestive function [Zhu 2019]. Second, there is no $\beta$-lactoglobulin in breast milk; thus, allergies are not easily caused [Hochwallner 2014; Fiocchi 2011]. Third, there are no additive ingredients in breast milk that can increase osmotic pressure or cause damage to the gastrointestinal mucosa. In short, all of the above advantages can help shorten the time of achieving total enteral nutrition in breast-fed infants. In this study, the time of achieved total enteral nutrition in the breast milk feeding group was an average of 2.2 days faster than that in the formula milk feeding group.

Compared with formula milk feeding, breast milk feeding can better reduce the intolerance rate of feeding, help to 
absorb nutrients and accelerate the growth and development of infants. Studies have shown that feeding intolerance might be related to gastrointestinal infection, immature development, improper feeding, flora disorders, and other factors [Eveleens 2020]. There is a large amount of osteopontin in breast milk, which plays an important role in the anti-inflammatory response, inflammatory cell chemotaxis and immune response, and oligosaccharides in breast milk can effectively eliminate intestinal pathogenic bacteria [Manthe 2019; Froh 2014]. This study showed that the incidence of complications, such as the intolerance rate of feeding, anemia, dyspeptic diarrhea and nosocomial infections, was lower in the breast milk feeding group than in the formula milk feeding group. The subjects in this study were infants undergoing cardiac surgery with cardiopulmonary bypass and hypothermia, so the patients may have had varying degrees of circulatory and gastrointestinal insufficiency. These advantages of breast milk feeding are more conducive to the postoperative recovery of these patients.

Malnutrition increases the incidence of complications and the health care-related costs of hospitalized patients. For infants after cardiac surgery, malnutrition means a longer feeding time and hospital stay, higher hospital costs, and mental burden on their families. The results of Fitria et al showed that although the prealbumin level was more specific in predicting the inflammatory response, the bivariate model showed a positive correlation between the prealbumin level and nutritional outcome [Fitria 2019]. In this study, the levels of prealbumin and albumin in the two groups were similar before the operation, and the postoperative levels of prealbumin and albumin in the breast milk feeding group were better than those of the formula milk feeding group, which showed that the nutritional status of breast milk feeding after cardiac surgery was better and recovered more quickly.

Our research had some limitations. The sample size included in this study was relatively small and limited to infants after cardiac surgery, so it might be unreasonable for our results to be applied to other postoperative patient groups. This was not a randomized, double-blind, controlled study. Our data collection might have been biased, there were relatively few indicators adopted, and the data might have been biased in the recording process, which could affect the accuracy of the results. Future research must take into account many factors and include a larger sample size and longer follow-up duration to further confirm our conclusions.

\section{CONCLUSION}

Breast milk feeding is effective and safe in infants after cardiac surgery. Compared with formula milk feeding, breast milk feeding in these infants can improve feeding tolerance, quickly increase body weight, improve nutritional status, reduce the incidence of related gastrointestinal complications, and shorten the length of hospital stay.

\section{ACKNOWLEDGEMENT}

We highly acknowledge the contribution by the participants: Yi-Rong Zheng, Jing Wang, Ling-Shan Yu, Qiu-Yu Chen, Li-Li Chen, Ya-Li Huang.

\section{REFERENCES}

Andreas NJ, Kampmann B, Mehring Le-Doare K. 2015. Human breast milk: A review on its composition and bioactivity. Early Hum Dev. Nov;91(11):629-35

Boban M, Persic V, Miletic B, Kovacicek K, Madzar Z. 2013. Heart Surgery Stems Increased Nutritional Risk, Expressed During the Course of Stationary Rehabilitation. Ann Nutr Metab. 63(1-2):17-24.

El-Alameey IR, Ahmed HH, Monir ZM, Rabah TM, Abdel Gawad AM. 2015. Predictors of High Serum Casein Antibody Levels Among Malnourished Infants and Young infants With Congenital Heart Disease. Open Access Maced J Med Sci. Mar 15;3(1):91-8.

Eveleens RD, Joosten KFM, de Koning BAE, Hulst JM, Verbruggen SCAT. 2020. Definitions, predictors and outcomes of feeding intolerance in critically ill infants: A systematic review. Clin Nutr. Mar;39(3):685-693.

Fiocchi A, Bouygue GR, Albarini M, Restani P. 2011. Molecular Diagnosis of Cow's Milk Allergy. Curr Opin Allergy Clin Immunol. Jun; 11 (3): 216-21.

Fitria L, Caesa P, Joe J, Marwali EM. 2019. Did Malnutrition Affect Post-Operative Somatic Growth in Pediatric Patients Undergoing Surgical Procedures for Congenital Heart Disease? Pediatr Cardiol. Feb;40(2):431-436.

Froh EB, Spatz DL. 2014. An Ethical Case for the Provision of Human Milk in the NICU. Adv Neonatal Care. Aug;14(4):269-73.

Glavin K, Roelants M, Strand BH, Júlíusson PB, Lie KK, Helseth S, Hovengen R. 2014. Important periods of weight development in childhood: a population-based longitudinal study. BMC Public Health. Feb $13 ; 14: 160$.

Goldberg JF. 2015. Long-term Follow-up of "Simple" Lesions--Atrial Septal Defect, Ventricular Septal Defect, and Coarctation of the Aorta. Congenit Heart Dis. Sep-Oct 10(5):466-74.

Hassiotou F, Geddes D. 2013. Anatomy of the human mammary gland: Current status of knowledge. Clin Anat. 26(1):29-48.

Hinde K, German JB. 2012. Food in an Evolutionary Context: Insights From Mother's Milk. J Sci Food Agric. Aug 30;92(11):2219-23.

Hochwallner H, Schulmeister U, Swoboda I, Spitzauer S, Valenta R. 2014. Cow's milk allergy: From allergens to new forms of diagnosis, therapy and prevention. Methods. Mar 1; 66(1): 22-33.

Khoshnood B, Lelong N, Houyel L, Thieulin AC, Jouannic JM, Magnier S, Delezoide AL, Magny JF, Rambaud C, Bonnet D, Goffinet F. 2012. EPICARD Study Group. Prevalence, Timing of Diagnosis and Mortality of Newborns With Congenital Heart Defects: A Population-Based Study. Heart. Nov;98(22):1667-73.

Kreissl A, Sauerzapf E, Repa A, Binder C, Thanhaeuser M, Jilma B, Ristl R, Berger A, Haiden N. 2017. Starting Enteral Nutrition With Preterm Single Donor Milk Instead of Formula Affects Time to Full Enteral Feeding in Very Low Birthweight Infants. Acta Paediatr. Sep;106(9):1460-1467.

Liu S, Joseph KS, Lisonkova S, Rouleau J, Van den Hof M, Sauve R, 
Kramer MS. 2013. Canadian Perinatal Surveillance System (Public Health Agency of Canada). Association Between Maternal Chronic Conditions and Congenital Heart Defects: A Population-Based Cohort Study. Circulation. Aug 6;128(6):583-9.

Manthe ED, Perks PH, Swanson JR. 2019. Team-Based Implementation of an Exclusive Human Milk Diet. Adv Neonatal Care. Dec;19(6):460-467.

Prieto MB, López-Herce J. 2011. Malnutrition in the Critically Ill Child: The Importance of Enteral Nutrition. Int J Environ Res Public Health. Nov;8(11):4353-66.

Radman M, Mack R, Barnoya J, Castañeda A, Rosales M, Azakie A, Mehta N, Keller R, Datar S, Oishi P, Fineman J. 2014. The Effect of Preoperative Nutritional Status on Postoperative Outcomes in infants Undergoing Surgery for Congenital Heart Defects in San Francisco (UCSF) and Guatemala City (UNICAR). J Thorac Cardiovasc Surg. Jan;147(1):442-50.
San Agustin JT, Klena N, Granath K, Panigrahy A, Stewart E, Devine W, Strittmatter L, Jonassen JA, Liu X, Lo CW, Pazour GJ. 2016. Genetic link between renal birth defects and congenital heart disease [J]. Nat Commun 7:1103.

Venugopalan P, Akinbami FO, Al-Hinai KM, Agarwal AK. 2001. Malnutrition in infants with congenital heart defects. Saudi Med J. 22(11):964-967.

Wren C, Irving CA, Griffiths JA, O'Sullivan JJ, Chaudhari MP, Haynes SR, Smith JH, Hamilton JRL, Hasan A. 2012. Mortality in Infants With Cardiovascular Malformations. Eur J Pediatr. Feb;171(2):281-7.

Yun SW. 2011. Congenital Heart Disease in the Newborn Requiring Early Intervention. Korean J Pediatr. May;54(5):183-91.

Zaidi S, Brueckner M. 2017. Genetics and Genomics of Congenital Heart Disease. Circ Res. Mar 17;120(6):923-940.

Zhu J and Dingess KA. 2019. The Functional Power of the Human Milk Proteome. Nutrients. Aug; 11(8): 1834. 